\title{
EFFECT OF DOUBLY SELECTIVE CHANNELS ABOUT BIT ERROR PROBABILITY IN OFDM AND FBMC
}

\author{
Bircan Kamislioglu*1, Ayhan Akbal ${ }^{2}$ \\ ${ }^{1,2}$ Firat University \\ *Corresponding author; bkamislioglu@ firat.edu.tr
}

\begin{abstract}
Filter bank multi carrier (FBMC) shows better spectral properties compared orthogonal frequency division multiplexing (OFDM). Double selective channels are described time selectivity and frequency selectivity which explore their performance variance in this paper. Closed form bit error probability (BEP) derives one top equalizers linear modulation techniques with OFDM and FBMC structures. When interference is closed to Gaussian noise, BEP error researching and Monte Carlo simulations are validated our analytically calculations. This paper presents us three scenarios that are OFDM without cyclic prefix (CP), CP-OFDM and FBMC applications about BEP over signal to noise ratio (SNR) for different channel models and different velocities. Applications are realized for theoretical, Gaussian approach, simulations and variations are shown with figures. The obtained $B E P$ expressions utilize to define time variant propagation for OFDM and FBMC.

Key words: OFDM, FBMC, Channel models, BEP, CP, Signal to noise ratio, time varying channels.
\end{abstract}

\section{Introduction}

In next generation wireless system, high data rates and low latency transmission have to been support because there is flexible allocation in time and frequency resources. But this requires are not possible in OFDM due to its bad spectral properties. On the contrary OFDM, FBMC is known an efficient alternative due to better spectral behaviors [1]. This paper purposes FBMC based Offset Quadrature Amplitude Nodulation (OQAM) due to achieving maximum spectral efficiency. But when we consider Multiple Input Multiple Output (MIMO) systems in FBMC is not simple as in OFDM, because of the intrinsic imaginary interference and an available choice is obtained for future wireless systems with an efficient application of MIMO [2]. We assume that there is a comparison in different modulation layout with selectivity of time and frequency that is double selectivity. BEP values are a significant criterion for comparing different modulation techniques. In literature, the BEP in OFDM is researched by many researchers for time variant and double selective channel [3, 4]. BEP is known a more intuitive metric that researchers used different interpolation methods [5-7] such as linear, spline, low pass (BER). However, the comparisons realized only on simulations, there are no analytical solutions. So a closed form structure of BEP with channel estimation is improved for linear 
interpolation methods. BEP in doubly selective channels is proposed for arbitrary linear modulation methods upon one tap equalizers such as FBMC and OFDM in this paper.

In Section 2 system model is explained, BEP formulation is given in Section 3, numerical results are shown in Section 4 and we finish this paper with conclusion.

\section{System Model}

Multi carrier modulation obtains transmission system that symbols are transmitted in a rectangular time frequency framework.

In transmission system, $x_{l, k}$ data symbols at subcarrier position $l$ and time position $k$ are modulated with based pulse $g_{l, k}(t)$ and transmitted signal $s(t)$ can be formulated as Equation 1 . We assume that $x_{l, k}$ is symbol of alphabet of QAM or PAM.

$$
s(t)=\sum_{k=0}^{K-1} \sum_{l=0}^{L-1} g_{l, k}(t) x_{l, k}
$$

Basis pulses $g_{l, k}$ also formulated as Equation 2, a time and frequency shifted sample of prototype filter $p(t)$ which is based on Hermite polynomials showed in Equation 3.

$$
\begin{aligned}
& g_{l, k}(t)=p(t-k T) e^{j 2 \pi l F(t-k T)} e^{j \frac{\pi}{2}(l+k)} \\
& p(t)=\frac{1}{T_{0}} e^{-2 \pi\left(\frac{t}{T_{0}}\right)^{2}} \sum_{\substack{i=\{0,4,8 \\
12,16,20\}}} a_{i} H_{i}\left(2 \sqrt{\pi} \frac{t}{T_{0}}\right)
\end{aligned}
$$

In Equation 2, time spacing $T$ and frequency spacing $F$ are used for defining spectral efficiency. In Equation 3, when the basis pulses for in $T=T_{0}$ time spaces and frequency spacing in $F=2 / T_{0}$. While maximum spectral efficiency is obtained for $T F=1$ that is not relevant for placed pulses in time and frequency domain [1]. FBMC only transmits real symbols so only real orthogonality is satisfied. Transmit signal is rewritten as Equation 4.

$$
s=G x
$$

Received data symbols are formulated in Equation 5. Channel matrix is indicated with $h$, complex Gaussian noise vector is represented with $n$. Also $D$ is formulated as Equation 6.

$$
\begin{aligned}
& y=\operatorname{diag}\{h\} D x+n \\
& D=G^{H} G
\end{aligned}
$$




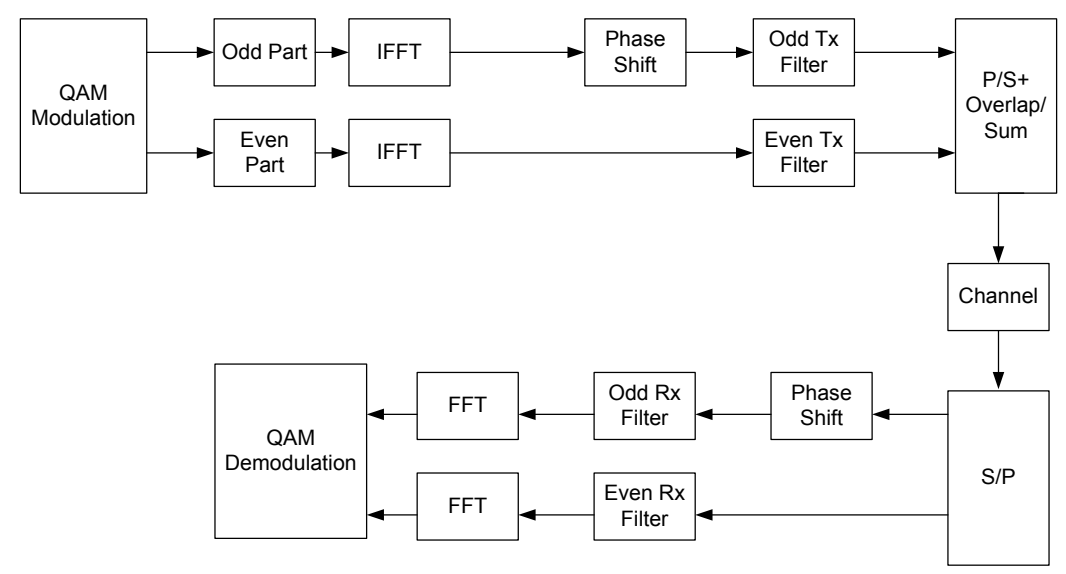

Figure 1. QAM-FBMC block diagram

$D$ indicates identity matrix in OFDM while in FBMC $D$ has non diagonal imaginary terms. Data symbols divide received symbols at the pilot positions in OFDM and the D is identity matrix. The channel values obtained with interpolation at the data positions. But FBMC consist of nandiagonal imaginary interference terms so FBMC is considered real part of symbol to cancel imaginary interference. However, FBMC system succeeds after equalization of the phase shift caused by the channel. Because this phase shift is not known before channel estimation complex domain is used the instead of the real domain. Since the interference is only available in imaginary valued symbols, interference will be completely eliminated using the real part of symbol. But Signal-to-Interference Ratio (SIR) is $0 \mathrm{~dB}$ in the complex domain and this is obviously too low in an exact channel estimation. To use channel estimation in FBMC with pilot-symbol, we thus have to cancel the imaginary interference.

\section{Bit Error Probability}

Closed form BEP is utilized from Lemma formulation [8]. Assuming $X$ and $Y$ are zero mean correlated complex valued Gaussian random variables and complex Gaussian ratio $X / Y$ is smaller than an exact value $Z_{R}$.

$$
P_{r}\left(R\left\{\frac{X}{Y}\right\}<Z_{R}\right)
$$

Obtaining compact expression for BEP two interpolation methods are important because the methods are not dependent of particular time and frequency positions that are Nearest Neighbor Interpolation and MMSE Interpolation (especially for flat fading channel). We search issue of finding the optimal power allocation by minimizing BEP, so that we describe the pilot to data power offset in Equation 8 .

$$
\kappa=\frac{P_{P}}{P_{D}}
$$




\section{Numerical Results}

We realized that doubly flat fading obtains a significant situation due to describing the BEP for a doubly selective channel when the SNR has smaller value than threshold value. BEP to SNR is illustrated in Figure 2 for doubly selective channel. The simulations performance is not dependent to specific modulation scheme [9].

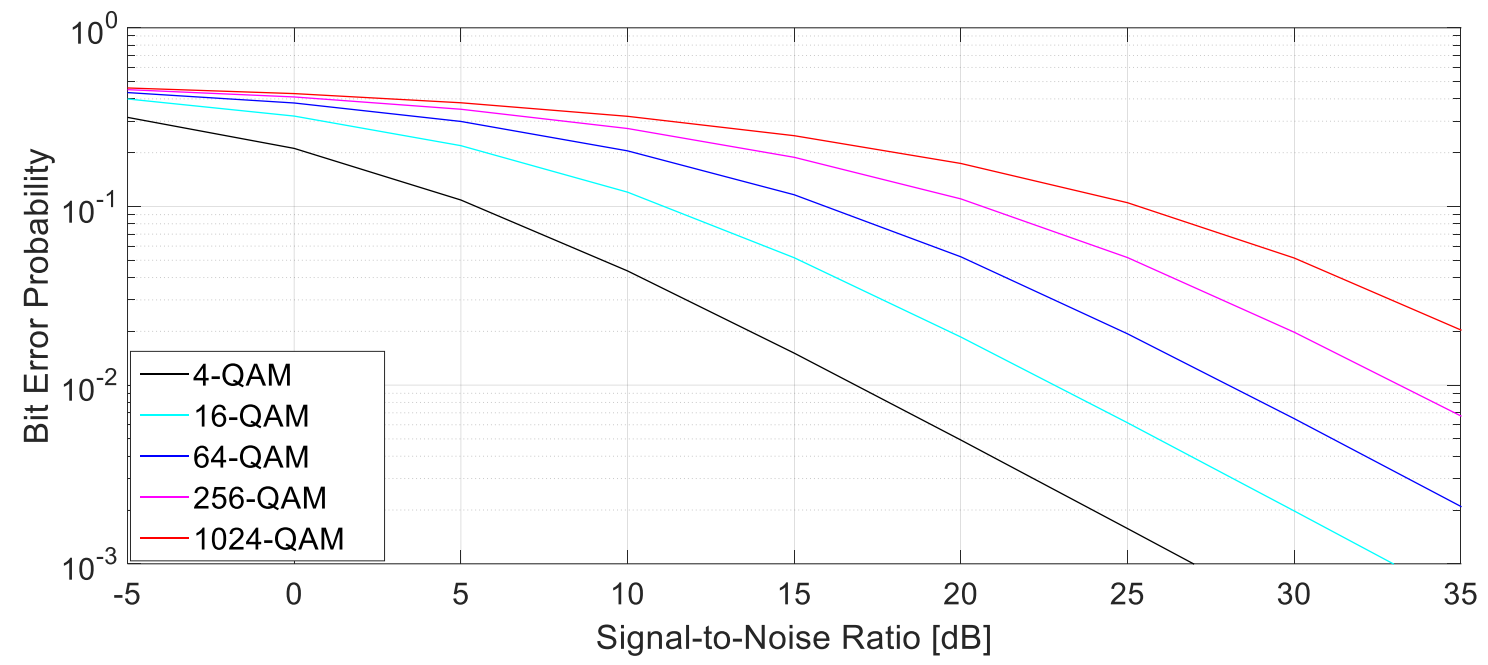

Figure 2. BEP to SNR for different modulation orders

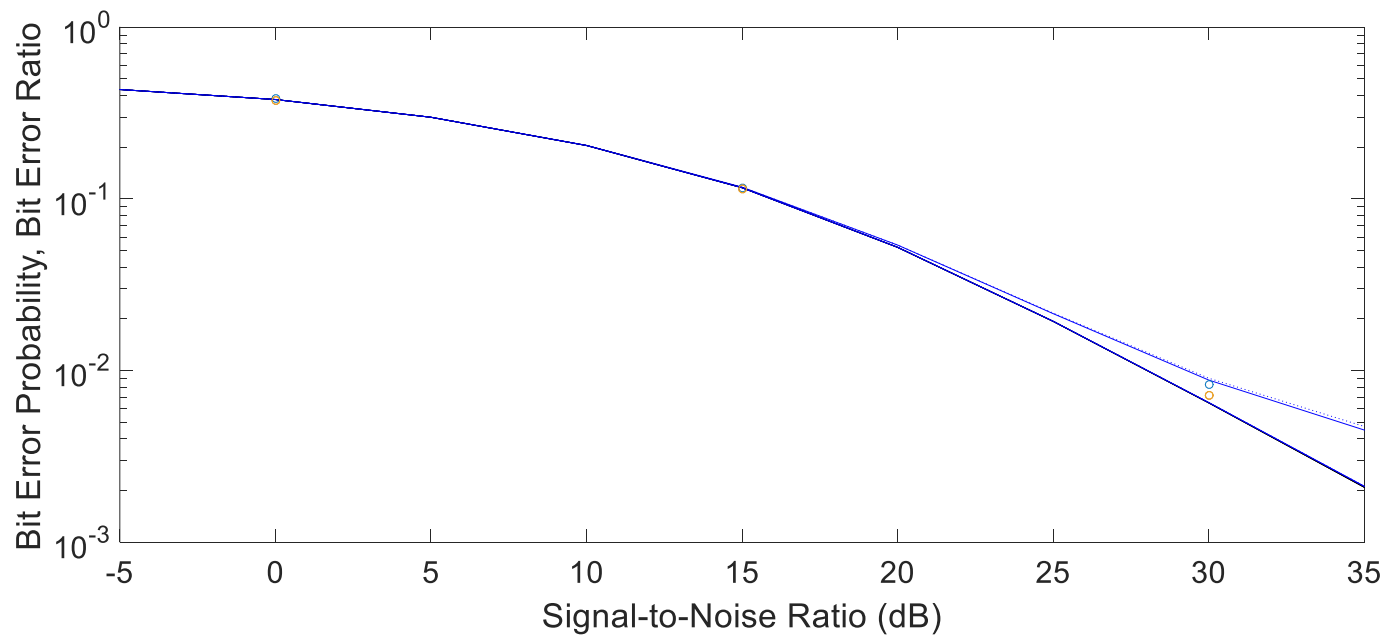

Figure 3. BEP to SNR for Pedestrian A channel model

In Figure 3, we used Pedestrian A channel model and the channel utilizes from Root Mean Square (RMS) in $46 \mathrm{~ns}$ delay spread. When we compare much higher delay spread channels and the small delay spread which obtains reality to current mobile communication systems [10]. 


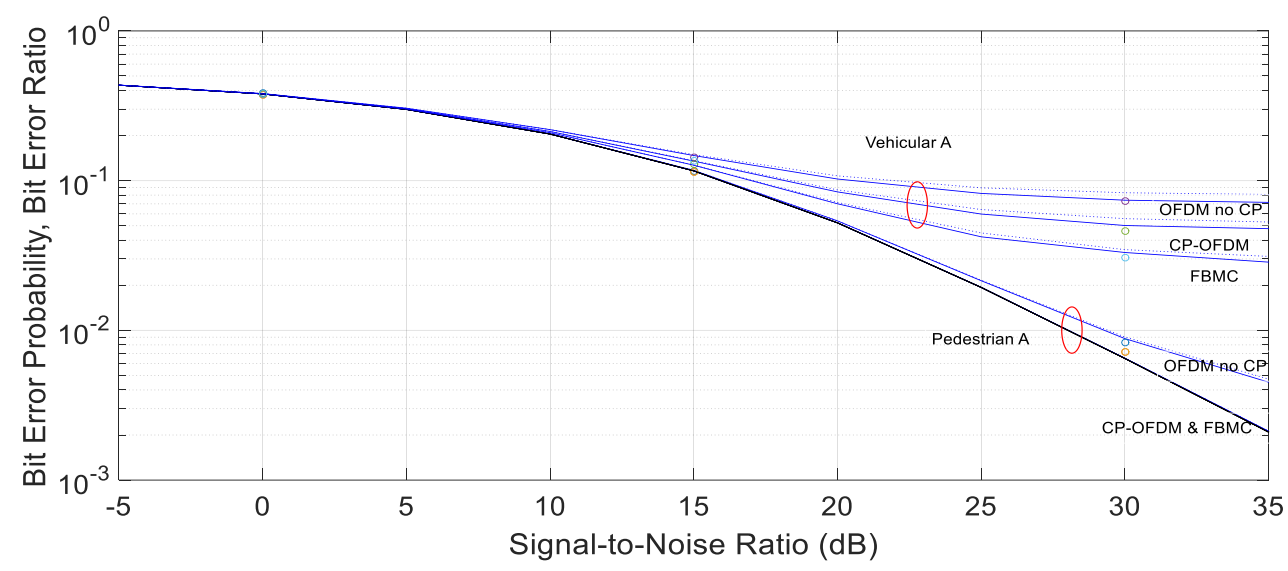

Figure 4. BEP to SNR for Pedestrian A channel model and Vehicular A channel model

In Figure 4 Pedestrian A channel model and Vehicular A channel model are used and results of simulations made accurate our calculations with Equation 7. In our work we used 64-QAM symbols, theory of BEP is showed with continuous line, Gauss approximation is showed with dashed line and simulation results is showed circle symbols. The Vehicular A channel model has high RMS delay spread with $370 \mathrm{~ns}$.

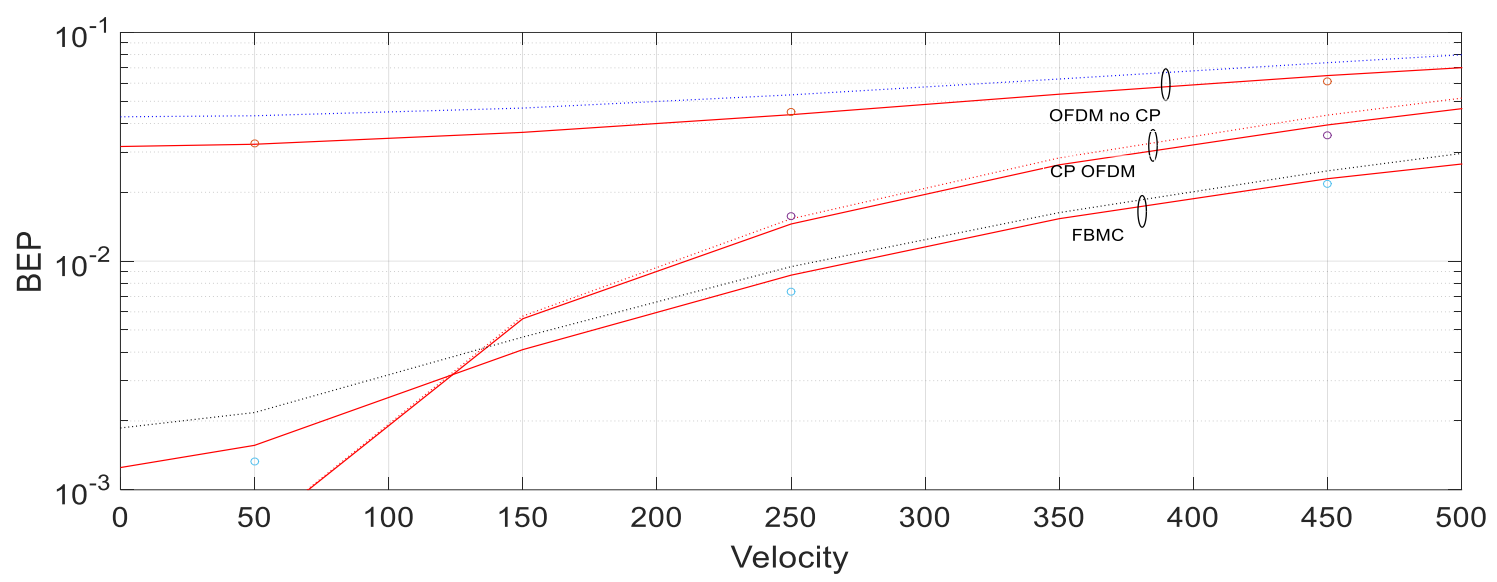

Figure 5. BEP to Velocity for Vehicular A channel model

BEP depended on velocity is showed in Figure 5 with zero noise and Vehicular A channel model. Figure 5 shows CP-OFDM has the lowest BEP for low velocities because of frequency selectivity comes from interference and in lower bit rate, we can perfectly eliminate. FBMC is showed better robustness in time variant channels so FBMC outperforms CP-OFDM for higher than $100 \mathrm{~km} / \mathrm{h}$ velocities.

\section{Conclusion}

This paper presents us to research the effect of time variant propagation in FBMC and OFDM with BEP expressions. FBMC shows better performance than CP-OFDM because of prototype filter better placed on time-frequency domains. However interference in high SNR affects both methods badly. 
CP-OFDM outperforms FBMC only in highly frequency selective channel. In this work analytic and simulation results supports each other. In future works we can improve FBMC in highly frequency selective channels and in different channel models.

\section{References}

[1] Farhang-Boroujeny, B., OFDM versus filter bank multicarrier, IEEE Signal Process. Mag., 28 (2011), 3, pp. 92-112.

[2] Nissel, R., Rupp, M., Enabling low complexity MIMO in FBMC OQAM, IEEE Globecom Workshops (GC Wkshps), 2016, pp. 1-6.

[3] Rugini, L., Banelli, P., BER of OFDM systems impaired by carrier frequency offset in multipath fading channels, IEEE Trans. WirelessCommun., 4(2005), 5, pp. 2279-2288.

[4] Nissel, R., Lerch, M., Simko, M., Rupp, M., Bit error probability for pilot symbol aided OFDM channel estimation in double selective channels, WSA, 2014.

[5] Dong, X., Lu, W.-S., Soong, A., Linear interpolation in pilot symbol assisted channel estimation for OFDM, IEEE Transactions on Wireless Communications, 6(2007), 5, pp. 1910-1920.

[6] Hsieh M.-H., Wei, C.H., Channel estimation for OFDM systems based on comb-type pilot arrangement in frequency selective fading channels, IEEE Transactions on Consumer Electronics, 44(1998), 1, pp. 217-225.

[7] Coleri, S., Ergen, M., Puri, A., Bahai, A., Channel estimation techniques based on pilot arrangement in OFDM systems, IEEE Trans. Broadcasting, 48(2002), pp. 223-229.

[8] Nissel, R., Caban, S., Rupp, M., Closed form capacity expression for low complexity BICM with uniform inputs, IEEE International Symposium on Personal, Indoor and Mobile Radio Communications (PIMRC), Hong Kong, China, 2015.

[9] Nissel, R., Rupp, M., OFDM and FBMC-OQAM in doubly-selective channels: calculating the bit error probability, IEEE Communications Letters, 21(6), 2017.

[10] Asplund, H., Larsson, K., and Okvist P., How typical is the 'typical urban' channel model? Proc. IEEE VTC, 2008, pp. 340-343. 